\title{
Céline Lesourd
}

\section{«CAPITAL BEAUtÉ». DE QUELQUES RICHES}

\section{FEMMES MAURES}

\begin{abstract}
«ÊTRE BELLE » EN PAYS MAURE, C'EST faIRE LE PRESTIGE DE SON GROUPE. MAIS UN CHARMANT SOURIRE, UNE GESTUELLE EN DENTELLE, UN EMBONPOINT VOLUBILE ET LA RÉPLIQUE COQUETTE PEUVENT ÉGALEMENT FOURNIR À QUELQUES FEMMES L'OCCASION DE SE DISTINGUER ET D'ACCÉDER AUX MOYENS DE LEURS AMBITIONS. LA BEAUTÉ PEUT CONDUIRE À UN BEAU MARIAGE - AVEC UN HOMME RICHE OU DE BONNE TENTE - OU À DES RELATIONS PLUS BRÈVES MAIS LUCRATIVES. EN CONVERTISSANT AINSI LEUR CAPITAL BEAUTÉ EN CAPITAL RELATIONNEL ET FINANCIER, CERTAINES FEMMES SONT DEVENUES DE GRANDES COMMERÇANTES, DES DAMES ÉVOLUANT DANS LES SPHÈRES POLITICO-COMMERCIALES.
\end{abstract}

Des campements de la bâdiyya ${ }^{1}$ à la capitale mauritanienne, d'hier à aujourd'hui, dans la société maure, hommes et femmes incarnent l'honneur et le prestige du groupe. Les hommes doivent faire preuve d'audace, de bravoure, d'intelligence, de force - $c^{\prime}$ est leur comportement qui est important; les femmes, quant à elles, matérialisent physiquement l'honneur et le prestige. La richesse et la dignité s'inscrivent dans leur chair. Ainsi, le corps des femmes - de certaines femmes du moins - est le corps du groupe. «Éternelles prestations sociales ${ }^{2}$ », les Dames doivent avant tout être belles: corpulente hier mais aujourd'hui plus mince, une riche Baydhâniyya ${ }^{3}$ doit être parée de bijoux précieux, couverte d'un voile luxueux et distiller sur son passage la fragrance des meilleurs encens ou parfums parisiens. Hier inactive sous la tente, elle sillonne aujourd'hui la ville à bord d'une décapotable ou d'un $4 \times 4$ fraîchement dédouané. Le corps féminin et ses parures mettent en scène les biens accumulés et une position sur l'échelle statutaire. Dans cette dictature de l'image de soi pour les siens, au nom du prestige et de l'honneur, l'enjeu consiste pour le groupe à inspirer le respect, la déférence et à tenir son rang 4 .

Cependant cette exigence d'embellissement constitue également un capital pour les femmes. L'importance accordée à l'esthétique ne répond pas uniquement à la nécessaire domestication féminine ni aux impératifs hiérarchiques, mais elle assure également la construction d'une certaine indé- 
pendance féminine. Si l'incorporation des valeurs sert le groupe, elle permet donc aussi à certaines femmes de se ménager des situations de pouvoir. En effet, les Baydhâniyyat ne sont pas moins socialement préparées à être séduisantes que séductrices : un sourire splendide, une répartie éclatante font leur succès et les compétitions masculines qu'elles attisent peuvent renverser les rapports de genre, voire même poser les Dames en situation de pouvoir symbolique, social et financier. Mieux, quelques femmes, grâce à leurs atours, sont parvenues à gravir l'échelle sociale, à se hisser sur le tremplin du messrah 5 , à briguer ainsi de véritables positions d'influence, tant au niveau économique que politique.

“OH, MIROIR, LAQUELLE DE NOS FEMMES EST LA PLUS BELLE ? "

Être femme d'un clan important nécessite l'incorporation d'un savant et paradoxal enseignement d'une culture de l'attitude féminine qui contribue à créer et reproduire l'échelle sociale. Dès lors, être et devenir femme, c'est marquer l'identité d'un groupe et son prestige:

"Le sentiment d'orgueil [est] au cœur de la asabiyya ${ }^{6}$ [qui] se manifeste dans le domaine de la défense et de l'intégrité sexuelle et physique des membres du groupe. $C^{\prime}$ est dans ce domaine que se nouent les rapports sociaux qui mettent en œuvre la compétition et qui perpétuent ou transforment les positions statutaires ${ }^{7}$.»

L'honneur des femmes maures, leur beauté et leur culture font partie intégrante du patrimoine du groupe. Du gavage - pratique jugée aujourd'hui

1. Zone de parcours des éleveurs nomades et des troupeaux. Terme que l'on peut traduire par la «brousse».

2. J. Baudrillard, Pour une Critique de l'économie politique du signe, Paris, Gallimard, 1972. Voir aussi J. Baudrillard, De la Séduction, Paris, Galilée, 1979.

3. Les Maures se désignent par le terme arabe baydhân, qui renvoie à la couleur blanche. Par extension, baydhân désigne les individus de culture baydhân, c'est-à-dire toutes personnes élevées dans le contexte de al-kham al-baydhân, que l'on pourrait traduire par : «la langue des Blancs », soit le hassâniyya. Les Français les désignent par le terme de " Maures », les Wolofs et les Peuls les nomment: Nar (le feu/l'arrogant). Baydhân s'oppose à Sûdan qui en hassâniyya signifie « noir ». Baydhân renvoie également aux personnes libres (harr), par opposition aux esclaves ('abid) et aux affranchis (harâtîn). Baydhâniyya (au féminin): une femme maure. Il ne sera pas fait cas ici des autres Mauritaniennes (peules, wolofs, soninké).

4. Dans le Trab al-baydhân (pays des Maures) comme ailleurs, la sexualité, qui «permet de témoigner des relations sociales » constitue un enjeu majeur: voir M. Godelier, "Sexualité, parenté et pouvoir », La Recherche, $\mathrm{n}^{\circ} 213,1989, \mathrm{p}$. 1141. Offrir une femme, c'est préserver un statut, accéder à un puits et de nouveaux pâturages, élargir des alliances, renforcer numériquement un groupe ou bénéficier d'une protection. Des femmes, de leurs charmes et leur honneur, dépend donc la perpétuation des groupes. 5. Dans le contexte nouakchottois, ce terme fait référence à la jet set, nous y reviendrons.

6. Solidarité sociale d'essence agnatique et tribale.

7. P. Bonte, «Manière de dire ou manière de faire: peut-on parler d'un mariage arabe? », in P. Bonte (dir), Épouser au plus proche. Inceste, prohibitions et stratégies matrimoniales autour de la Méditerranée, Paris, EHESS, 1994, p. 390. 
révolue - aux cours de stretching dans un club privé, il convient pour affirmer et perpétuer une hiérarchie, d'hier à aujourd'hui, de façonner le corps féminin et d'ajuster sa parure.

\section{Sculpter}

Les jeunes filles, comme les petits garçons, se voient attribuer un nom le septième jour suivant leur naissance, lors d'une cérémonie ritualisée à laquelle n'assistent pas les hommes mariés et les anciens. Ce même jour, beaucoup sont excisées ${ }^{8}$. Il s'agit de contrôler une sexualité féminine pensée comme éruptive, incontrôlable, qui pourrait menacer l'honneur du groupe; d'ailleurs, en hassâniyya le terme darze désigne tant le sexe féminin que l'honneur. C'est bien sûr la honte de l'enfant né hors mariage qui est crainte ici, comme l'appuient les nombreuses expressions désignant les femmes qui ont donné vie à un bâtard (verkh): «elle a fait un enfant dans le désert», «elle a prié vers le Nord ${ }^{9} »-$ le Nord et le désert étant les espaces dans lesquels errent les mauvais génies (jnûn).

Très vite, les petites filles sont assignées aux (non)-activités féminines définies par leur position dans la hiérarchie sociale. Les plus nobles et les plus riches d'entre elles doivent observer une oisiveté totale. Traditionnellement, sous les «bonnes tentes», dans les familles nobles ou aisées, la fillette à peine âgée $\mathrm{d}^{\prime}$ une dizaine $\mathrm{d}^{\prime}$ années subissait ${ }^{10}$ le gavage (blûh), moment de $l^{\prime}$ inculcation de $l^{\prime}$ identité féminine ${ }^{10}$ :

«Maintenue par une vieille servante, l'infortunée Toutou se débattait [...]. Que faisait donc cet homme agenouillé à ses pieds? [...] Rampant vers le côté de la tente, je m'aperçus qu'il serrait les orteils de la pauvre petite entre deux bâtons à chameaux. Cela devait être une cruelle torture! Chaque fois que la victime ouvrait la bouche pour protester, $\mathrm{l}^{\prime}$ homme [...] saisissait une grande calebasse et faisait boire de force la pauvre Toutou. Elle bavait, pleurait, serrait les dents ; son tortionnaire recommençait à la battre, à lui broyer les pieds et les mains ${ }^{11}$.»

Pendant les mois de l'hivernage, entre août et octobre, chaque nuit, la jeune fille ingurgitait 15 à 20 litres de lait de vache ou de chamelle, parfois accompagnés de calebasses de beurre fondu et de gâteaux ('aysh), sous la menace de sa mère ou d'un(e) forgeron(ne). Cette pratique, aujourd'hui considérée par beaucoup comme désuète, visait à affirmer l'importance du troupeau de la famille et donc à matérialiser son aisance matérielle.

«Au début la patiente se débat. Son estomac se révolte et elle éprouve des nausées [...]. Très vite, elle prend des proportions considérables, se gonfle et devient une masse de chair peu mobile et incapable de tout travail physique. Aussi cette pratique est-elle réservée à une infime partie de la société, aux classes bien pourvues de serviteurs, 
qui n'ont jamais besoin de chercher l'eau ou de préparer la nourriture. Les grosses femmes se déplacent assez difficilement et il leur faut l'aide de plusieurs personnes pour se hisser sur une selle ${ }^{12}$.»

Cet engraissement permettait également d'exercer un contrôle sur les femmes les plus prestigieuses, de limiter leurs déplacements et donc de s'assurer de leur bonne conduite: «Si elle a le ventre plein, elle ne voit rien ${ }^{13}$ ». L'incorporation de lait participait également, dans la continuité de l'ablation clitoridienne, à la canalisation des sexualités, au contrôle des désirs féminins : il ne fallait pas que la jeune fille soit «chaude» ou "salée».

Enfin, cette torture lactée s'accompagnait d'une socialisation de la femme, période d'apprentissage de l'importance d'être ravissante. Car il s'agit bien là d'un véritable travail d'embellissement: «Toutou, ma joie, mon œil, bois pour être belle, belle comme la lune... Bois, ô ma tourterelle, ne déshonore pas ta vieille nourrice ${ }^{14}$ ». Une belle femme "tirait ses fesses derrière elle». Les proéminences devaient se situer sur le bas du corps : les mollets, les cuisses; les hanches devaient être charnues, en contraste avec une taille fine, un ventre plat, une poitrine presque absente. Afin que les rondeurs ne se forment pas anarchiquement, le corps de la fillette était donc massé pour que le lait se dépose sur les parties anatomiques souhaitées, où de profondes vergetures venaient tisser leur trame.

8. En Mauritanie, le taux de prévalence de cette pratique serait de $71 \%$; les femmes soninké seraient les plus touchées (90\% d'entre elles), puis les femmes maures, peules (70 \%) et enfin wolof (28\%). Voir A. Daddah, Diagnostic-bilan de la situation de la femme en Mauritanie, Nouakchott, Secrétariat à la condition féminine, 2003.

9. Expressions relevées dans A. Tauzin, Figures du féminin dans la société maure (Mauritanie), Paris, Karthala, 2001, p. 39-40.

10. Affectée par la sécheresse et l'urbanisation, mise à mal par la mode, réprouvée par l'État, décriée par la médecine, la pratique du gavage est largement tombée en désuétude. Elle est ici décrite grâce à des témoignages recueillis auprès de Nouakchottois ayant grandi à la bâdiyya et à des observations plus anciennes, celles d'O. du Puigaudeau parmi les Maures de Mauritanie dans les années 1930, Pieds nus à travers le désert, Paris, Plon, 1992 et d'E. Bernus, Touaregs nigériens. Unité culturelle et diversité régionale d'un peuple pasteur, Paris, Orstom, 1981, pour les Touaregs du Niger des années 1970. Voir également R. Popenoe, Feeding Desire: Fatness, Beauty and Sexuality among a Saharan People, Londres, Routledge, 2004 ; A. Tauzin, Figures du féminin..., op. cit.; C. Fortier, «Le corps comme mémoire: du giron maternel à la férule du maître coranique », Journal des Africanistes, vol. 68, n $1-2,1998$, p. 199-223 ; C. Fortier, «Corps, différences des sexes et infortune. Transmission de l'identité et des savoirs en Islam Malékite et dans la société maure de Mauritanie ", thèse de doctorat en anthropologie sociale, Paris, EHESS, 2000.

11. O. du Puigaudeau, Pieds nus..., op. cit., p. 139.

12. E. Bernus, Touaregs nigériens..., op. cit., p. 145.

13. Propos recueillis par A. Tauzin, «Sexualité, mariage et stratification sociale dans le Hodh mauritanien ", thèse de $3^{\text {e }}$ cycle en anthropologie, Paris, 1981.

14. O. du Puigaudeau, Pieds nus..., op. cit., p. 140. 
Si certains critères esthétiques demeurent toujours d'actualité - la peau doit être blanche et douce, dénuée de toute pilosité ; la dentition, d'une blancheur éclatante, doit contraster avec des gencives plus sombres (autrefois maquillées au kohl) ; dentelées, les incisives déterminent encore la qualité du sourire -, aujourd'hui, et plus particulièrement en milieu urbain, la mode s'est inversée et la minceur est de mise. L'idéal n'est plus d'être en chair mais d'affirmer sa volonté de mincir, de dire ses efforts pour garder la ligne. La prise de conscience des problèmes de santé liés au surpoids participe à cette évolution, mais celle-ci renvoie également à une sorte d'inversion des marqueurs des identités masculines et féminines. Alors qu'avant, comme l'a montré C. Fortier ${ }^{15}$, les hommes devaient paraître aussi minces que les femmes devaient être enrobées, aujourd'hui la corpulence des hommes témoigne de leur plus grande sédentarité et marque leur réussite ; la différence des formes participant à l'affirmation des limites des genres, le maintien de la dichotomie corporelle enjoint de facto aux femmes de maigrir. Toutefois, les femmes maures sont en réalité rarement minces. Elles perdent du poids et maintiennent leur ligne, certes, mais l'essentiel de cette quête ne consiste pas uniquement en un résultat physique : échapper à l'obésité, s'affiner ou rester fine requiert un changement d'alimentation et parfois même l'usage de traitements, de substituts alimentaires, de produits diététiques, et ces solutions ont un coût. Acheter à Nouakchott des produits «allégés » est fort onéreux, rapporter de voyages des compléments d'alimentation (soupes, laits, gélules... amincissants) constitue un véritable investissement, s'abonner à une salle de gymnastique privée demeure un luxe. Ainsi cette avancée sanitaire muée en nouveau chic urbain nécessite des dépenses élevées qui permettent, comme la «sur-en-chair» d'autrefois, d'exposer son statut social. Dire son effort de minceur, confier - en sachant que cela sera répété - son inscription au cours de stretching, laisser son véhicule devant le magasin de produits «naturels» et diététiques de Nouakchott sont autant de procédés ostentatoires utilisés pour marquer le statut social ${ }^{16}$.

\section{Orner}

Être belle, ou du moins le paraître et construire ainsi l'apologie de son groupe ou de celui de son mari... Tous ces efforts se lisent dans les trames parfumées des tissus de ces Dames. La malhafa ${ }^{17}$ est signifiante du statut auquel prétend celle qui le porte:

«Une femme riche peut porter le voile de tout le monde, dans les journées de tous les jours chez elle. Alors tu mets du gaze, ou du contre gaze. Des voiles pratiques, un peu vieux. Quand tu vas travailler, il faut mettre un beau tissu, un tissu neuf, un peu plus cher, dans les 4000 ouguiyas [khiatta]. Parfois tu peux mettre un ancien voile kenebo qui n'est plus à la mode [...] Il n'est plus à la mode car je l'ai porté deux fois, dans un 
salon et un mariage, je ne peux plus le remettre pour ces occasions, il faut en trouver d'autres, plus nouveaux pour les grands mariages [...]. Il y a des nouveaux qui arrivent toutes les semaines avec des commerçantes qui vont beaucoup à Dubaï [...]. Moi je ne suis pas assez riche pour avoir un voile kenebo neuf et à la mode à chaque soirée. J'en ai un en soie et en satin [50 000 ouguiyas] [...]. Les femmes très riches [...], elles vont dans toutes les soirées, dans les mariages importants, elles sortent beaucoup et elles portent toujours un voile différent [...]. Je ne manque pas d'argent, Alhamdulilah, mais je ne peux pas dépenser 200000 ouguiyas comme ça de voiles, tous les mois ${ }^{18}$ ! »

Avec ces techniques de soi(e), il faut sans cesse être la plus en vogue: les modes éphémères qui agitent la capitale, phénomène d'émulation et de compétition, participent pleinement à ce permanent souci hiérarchique de déclasser ou surclasser; parce que «l'objet est classant ${ }^{19}$ », la course au statut en appelle à toutes les « exhibitions » dont celles des modes qui régissent le commerce du voile et des téléphones. Ainsi, en soirée, une femme pourra porter une malhafa kenebo carmin aux imprimés légers jaunes pâles et verts d'eau; ses chaussures, rappelant la couleur des motifs du voile, sont aussi parfaitement associées à son sac à main. Cette coordination sans faute est rehaussée par l'éclat de bijoux en or. Une femme plus jeune, également fortunée, n'hésitera pas à ajouter du chic au chic en ajoutant un téléphone de couleur carmin à sa panoplie monochrome: le souci du détail est souvent très poussé car cette attention soutenue portée au corps et à ses outils est une technique de production de soi qui tend à instaurer une hiérarchie: «L'aristocrate semble dire par-là que lui et les autres ne sont pas faits du même bois 20 ».

Le téléphone portable est devenu un accessoire essentiel de ces luttes pour le classement. Les (re)vendeurs de mobiles interrogés distinguent nettement deux clientèles, l'une masculine, l'autre féminine, et plus l'on s'élève

15. C. Fortier, «Corps, différences des sexes...», doc. cit.

16. Les mises en scène de la richesse et le rôle que jouent les rumeurs dans la diffusion des réussites sont discutées dans C. Lesourd, «Au bonheur des Dames. Femmes d'affaires mauritaniennes de nos jours ", thèse de doctorat en anthropologie sociale, Paris, EHESS, 2006. Les entretiens et observations cités dans cet article ont été réalisés dans le cadre des terrains effectués entre 2002 et 2005 pour la préparation de la thèse, sauf pour ce qui concerne le voile porté par les femmes maures et l'insertion des Mauritaniennes en politique.

17. Voile que portent les femmes maures. «Simple», "gaze» et «contre gaze», «khiatta», «kenebo», «soie» : catégories de voile de 500 à 60000 ouguiyas, soit de moins de $2 €$ à $150 €$.

18. Propos tenus par une riche Nouakchottoise. L'entretien rapporté ici et les données relatives aux voiles sont le fruit d'un travail réalisé avec Olivier Schinz, doctorant en anthropologie à l'université de Neuchâtel, dans le cadre du tournage d'un documentaire en 2004.

19. P. Bourdieu, La Distinction. Critique sociale du jugement, Paris, Minuit, 1979.

20. J.-P. Warnier, Approches de la culture matérielle. Corps à corps avec l'objet, Paris, L'Harmattan, 1999, p. 110. 
sur l'échelle sociale, plus la différence est manifeste. Les hommes fortunés préfèrent les grands téléphones aux couleurs sobres, écran large, double clavier, stylet, connexion Internet et imprimante. Les femmes leur préfèrent des petits appareils à clapet, colorés, ornés de pacotilles. Elles arborent leur précieux gadget à la main, ou au cou. Ou alors, avec nonchalance, elles sortent et ressortent leur portable de leur sac, en feignant $d^{\prime} y$ chercher quelque chose. D'après les vendeurs, les femmes, et surtout les plus riches d'entre elles, constituent la meilleure des clientèles:

«Elles changent beaucoup de téléphone, parce qu'il leur faut toujours le plus nouveau, le plus cher [...]. Il y a des femmes qui viennent ici une fois par semaine, elles échangent un portable à 100000 ouguiyas [350€] qu'elles ont acheté la semaine dernière contre un autre encore plus cher. [...]. Elles rajoutent la différence.»

Interrogé sur la raison de ces perpétuels changements, un vendeur répond, amusé : «ce sont des batrounnes ${ }^{21}$, elles veulent être belles [...], elles veulent faire le théâtre [...], il faut toujours faire mieux que les autres femmes, c'est la concurrence».

\section{Enchanter}

Très tôt, les jeunes filles apprennent le code de la pudeur (sahwa), qui consiste en des attitudes de respect, de silence, d'humilité voire même de passivité. La retenue du langage et du corps est exigée des femmes devant les hommes auxquels elles doivent le respect - tous ceux qui font partie de la parenté interdite de mariage -, elles doivent être correctement couvertes de la malhafa - seuls le visage, les mains et les pieds peuvent apparaître hors du voile -, demeurer peu mobiles et rester silencieuses, sauf si leur avis est sollicité. En revanche, entre femmes, quels que soient l'âge et le statut, un tel comportement $\mathrm{n}^{\prime}$ est pas de rigueur ${ }^{22}$.

Paradoxalement, les fillettes, dès leur plus jeune âge, sont initiées aux arts de la coquetterie. Il ne s'agit donc pas uniquement d'un apprentissage de la pudeur, mais plutôt d'une double formation pudeur/séduction où les fillettes doivent savoir, en fonction des circonstances, user de l'un ou de l'autre de ces artifices ${ }^{23}$. Des mimiques enchanteresses sont enseignées dans l'univers féminin : la mère de l'enfant et ses proches parentes travaillent l'esprit de la jeune fille pour faire d'elle un être charmant et spirituel. Les exigences requises, au terme de cette initiation, sont nombreuses : audace, vivacité d'esprit, art de mener la conversation, assurance, humour, culture. Ces qualités ne doivent cependant pas être synonymes d'exubérance: les femmes doivent savoir user de pudeur, garante de leur bonne éducation, condition sine qua non pour que les «phases d'émancipation » soient socialement tolérées. 
«Les cousines Mariem et Aïchatou, âgées de 4 ans, vivent sous le même toit, chez leur grand-mère avec leurs tantes. Les femmes de cette grande famille sont réputées pour être des shabîbât ${ }^{24}$ et il semble que les jolies frimousses de Mariem et Aïchatou ne dérogent pas à la tradition.

Habillée dans une robe d'enfant assez courte, Aïchatou, en s'asseyant, étire ses froufrous pour essayer de les capturer entre ses doigts de pieds, comme le font ses aînées avec leur voile. Dans ce réflexe de pudeur tout de féminité, elle est encouragée joyeusement, de la même façon que lorsqu' elle part effrontément, avec sa cousine, à la conquête de toutes les chaussures à talons du haut desquelles elles défilent sous les youyous et les applaudissements de leurs tantes.

Très fréquemment ce défilé se poursuit par des séances de danse au cours desquelles les femmes chantent et battent des mains tout en enseignant aux petites filles les gestes "infaillibles". Aïchatou et Mariem se dandinent gracieusement en avançant, en reculant, elles lèvent légèrement les avant-bras et cassent et recassent leurs poignets. Leurs tantes les encouragent: "as-ski, as-ski" [joli, joli], puis ces petits bouts de femmes agitent les prunelles de leurs yeux, art dans lequel Aïchatou excelle. D'ailleurs, la petite fille sait bien que ce sera là le clou du spectacle qui lui vaudra tous les rires et les applaudissements de l'assemblée ${ }^{25}$. »

Ainsi, dans l'univers féminin, quand la pudeur n'est pas nécessaire, chaque expression, chaque rire et sourire, chaque geste de la main participe de cet art discret de plaire: replacer un pan de voile pour attirer le regard sur la chevelure et les avant-bras ; frapper la main de sa voisine après une remarque empreinte d'humour pour souligner une complicité. Sourires. Regards brillants. Jouer avec ses mains, les faire pivoter pour faire cliqueter ses bijoux; lever un doigt, puis deux, avec une délicate application. Hausser un peu la voix, la moduler. S'immobiliser. Froncer puis lever les sourcils et feindre la colère puis l'étonnement. Claquement de langue. Relever le menton. Chaque parcelle visible du corps (la malhafa glisse sans cesse) argumente le dialogue.

21. «Patron(ne)» (la lettre «p» n'existe pas en arabe, elle est souvent prononcée «b»), c'est ainsi qu'est désigné celui ou celle qui a du pouvoir et de l'argent. Entretien avec un employé d'une boutique de téléphone portable, Nouakchott, 2004.

22. À l'inverse, on attend des jeunes hommes qu'ils respectent leurs aînés ou leurs supérieurs en évitant certains sujets de conversation comme les femmes, la musique ou le tabac, mais aussi qu'ils fassent preuve de leur courage, de leur capacité de relever des défis entre égaux.

23. Ce code de la pudeur est imposé aux femmes en présence des hommes avec lesquels le mariage est prohibé. Précisons également que ces comportements sont plus ou moins marqués selon le groupe d'appartenance: une femme issue d'un groupe maraboutique ne peut ainsi se permettre la verdeur de langue d'une femme d'un groupe guerrier.

24. Shabîbât est le pluriel de shabîbâ, terme qui désigne une très belle femme dont le charme lui assure de faire la «une» des conversations et convoitises.

25. Notes de terrain, 2004. 


\section{JEUX DE DAMES}

Parce qu'elles représentent un capital matrimonial et symbolique, de fortes contraintes éducationnelles, physiques et morales pèsent sur les femmes. Mais au-delà de ces premières constatations, l'apprentissage d'être femme dans un corps de femme autorise une ambivalence au cœur des genres et des rapports de genre. Les hommes, courageux et forts, doivent parfois plier et céder aux caprices face aux Dames. Dès lors, dans ces sphères d'autonomie du monde féminin, le corps des femmes maures n'est plus seulement le corps du groupe. Il est un capital mobilisable dans les aventures financières, sociales et politiques des beautés les plus ambitieuses.

\section{De l'ambivalence dans les rapports de genre}

Durant la journée, les femmes demeurent essentiellement «à l'intérieur » (de la maison, de la boutique), où se succèdent repas, jeux, visites, travail et discussions ${ }^{26}$. En l'absence des hommes, les femmes maures, quelle que soit leur tranche d'âge, discutent librement puisque la sahwa n'est de rigueur qu'en présence masculine. Les voiles tombent, les conversations peuvent être parfois très directes quand il s'agit d'évoquer le sexe opposé. Il y a cependant toujours quelques hommes, profitant de l'absence de leurs congénères, qui font une visite impromptue. Cette cour masculine peut être nombreuse sous une même tente et certaines sont plus prisées que d'autres ${ }^{27} \ldots$

"C'est une maison de Nouakchott au centre de laquelle se tient une grande tente. Une vieille maison, une des plus anciennes de la capitale. Djemila, veuve, âgée et de bonne famille y vit avec ses filles et leurs cousins. Une maison de femmes emplie de rires, de discussions coquines, de regards évocateurs, de cris d'enfants. Une maison aux visites incessantes: quelques femmes, des amies, des parentes. Des jeunes, des vieilles. Mais surtout des hommes qui, toute la journée, viennent prendre le thé, fumer la pipe, s'entretenir avec la maîtresse de maison et ses filles. On y retrouve souvent le même préposé au thé. C'est Djemila qui le lui a suggéré. Il ne peut donc s'y soustraire. On retrouve là les mêmes hommes, concentrés face à cette vieille femme, adversaire invaincue au "jeu du 7" 28. Elle les observe de ses yeux si mobiles et brillants, assurée de sa victoire. Elle me sourit, parce qu' elle sait que je sais ce que tout le monde sait: Djemila triche effrontément. Et aux regards de tous, six paires d'yeux braqués sur elle, Djemila triche à nouveau.

Djemila est la maîtresse de maison, sa tente urbaine est une des tentes les plus prisées des Nouakchottois originaires de sa région, une de ces tentes qu'on aime à fréquenter: les femmes y sont belles, agréables et charmantes, les discussions s'enchaînent, parfois drôles, parfois nostalgiques. Djemila sait recevoir, sourire et faire rire. Même au "7", quand Djemila et l'assemblée des hommes veulent pimenter leurs échanges verbaux, elle les autorise à contester ses pratiques malhonnêtes. Ceci donne lieu à de longues altercations où chacun s'amuse, se cherche, sous l'orchestration victorieuse de leur hôtesse ${ }^{29}$.» 
Lors de ces rencontres quotidiennes, les femmes renversent la situation : elles se font faire le thé et obtiennent que l'un des hommes présents achète la menthe ou le sucre. Ces concessions masculines font l'objet d'une longue négociation durant laquelle les femmes dévoilent des trésors d'ingéniosité pour arriver à leur fin: de l'audace et un bon sens de la répartie parviennent à lever l'hésitation, parfois feinte, du visiteur. On assiste alors à des interactions de genres qui sont de l'ordre du rapport de séduction ${ }^{30}$. Mais c'est l'homme qui, en prenant place en «territoire féminin », choisit d'être l'instrument d'un jeu dont il n'est pas maître. Les femmes mènent la discussion, attirent, jouent, ce sont elles qui ont l'initiative de la parole; elles coupent court aux dires de leurs visiteurs, elles peuvent rire de lui, de sa gêne, de ses propos. Les femmes maures adoptent alors des archétypes comportementaux habituellement attribués aux hommes : elles ont la parole, l'initiative, elles usent des qualités masculines de force et de courage. De façon significative, elles ne se cachent plus sous leur voile, elles le laissent glisser pour découvrir légèrement les cheveux alors que le semi-découvert est un attribut connoté masculin, dans les oppositions binaires de genre. Elles n'hésitent pas non plus à se faire offrir des cigarettes et à les fumer avec nonchalance aux yeux de tous. Le contact physique peut devenir ensuite plus direct, et c'est l'homme qui en prend l'initiative s'il en a l'autorisation. Il peut jouer à saisir les mains ou les pieds de son interlocutrice, et parfois plus, dans des contextes plus intimes. Mais l'apparition d'un individu auquel l'on doit un comportement de sahwa peut soudainement changer les règles du jeu et replacer les individus dans une attitude où hommes et femmes doivent se conformer à l'image de leur sexe, imposée par les normes.

L'ambivalence se poursuit à la nuit tombée. Dans le quartier résidentiel de Tevragh-Zeïna, hommes et femmes disposant d'un véhicule circulent en ville suivant un itinéraire bien particulier; ils empruntent la «corniche», une

26. Notons que même sur certains lieux de travail, il est possible d'observer ce genre de scène.

27. Il ne faut évidemment pas que ceux-ci soient apparentés aux femmes, ni même qu'un des hommes doive sahwa à un autre.

28. Le « 7 » se joue avec des dés et un tapis sur lequel on avance des pions qu'il faut préserver de son adversaire, en suivant un parcours de retour à la case départ. Dans le même esprit que le jeu des petits chevaux.

29. Notes de terrain, juin 2004.

30. Au sujet des rapports de séduction dans la société maure, voir A. Tauzin, Figures du féminin..., op. cit.; C. Fortier, "Épreuves d'amour en Mauritanie», L'Autre, Cliniques, cultures et sociétés, vol. 4, $n^{\circ} 2,2003$, p. 239-252; du même auteur, «Séduction, jalousie et défi entre hommes. Chorégraphie des affects et des corps dans la société maure», in F. Héritier et M. Xanthakou (dir.), Corps et affects, Paris, Odile Jacob, 2004 ; O. Schinz, "Construction du genre chez les Maures de Mauritanie», Université de Neuchâtel, mémoire de licence en ethnologie, 2001. 
succession de routes goudronnées qui cheminent du stade olympique au carrefour du Bana Blanc, puis redescendent le long de l'avenue des ChampsÉlysées, pour ensuite bifurquer dans la rue des snacks, dite rue du Prince ${ }^{31}$. La promenade se dirige alors vers le café-restaurant huppé La Palmeraie, puis vers l'ambassade de France avant de revenir au stade olympique. Ce tour peut se répéter, en fonction du public en piste, et ce circuit, où les voitures croisent à faible allure (entre 30 et $50 \mathrm{~km}$ à l'heure), est quotidiennement surchargé dès 21 heures.

«Mercedes, Toyota, Nissan et Renault se suivent. Les 4x4, berlines, décapotables et pick-up se croisent et se recroisent. On peut reconnaître $X$ ou $Z$ à sa voiture et c'est bien ainsi: c'est le signe que cette voiture est visible, remarquable, à l'image de son propriétaire. Les jeunes hommes circulent avec des amis de leur classe d'âge, les véhicules de jeunes filles baladent cousines et copines. Des hommes circulent seuls, à la recherche de compagnons masculins ou de conquêtes féminines. Quelques couples avec leurs enfants, plus rares, se mêlent à cet étrange cortège de l'asphalte. On remarque également les tête-à-tête amoureux de jeunes mariés. Dans chaque véhicule, la relation entre chauffeur et passagers est lisible pour tous: dans le cas d'un jeune couple, par exemple, la jeune femme est enfoncée dans son siège, dans une position assez horizontale, mais elle se sert de sa portière pour s'adosser, elle a donc le regard rivé sur son conducteur. Cette attitude est parfaitement claire pour les usagers de la corniche. Pour peu que le jeune homme et la jeune femme soient reconnus, l'observateur pourra ainsi colporter la nouvelle: "un tel et une telle se sont mariés". Les hommes, entre eux, s'ils ne sont pas en situation de sahwa, écoutent de la musique, ils se vautrent dans leur siège et posent leurs pieds sur le tableau de bord.

Ces ballets automobiles répondent à deux motivations : la convivialité, d'une part, et la mise en scène - souvent ostentatoire - $d$ 'autre part. C'est un moment de rencontres où chacun se livre aux regards. Les célibataires s'aperçoivent, se croisent, se suivent. Le jeune homme reconnaît sa dulcinée à sa voiture, il la suit, essaie d'attirer son attention à un carrefour. Il peut également apercevoir qui sont les camarades de la jeune femme. Peut-être pourra-t-il, s'il connaît l'une d'elles, se procurer le numéro de portable de la Belle...

Ce sont généralement les femmes qui chorégraphient le parcours des cylindrées. Elles conduisent, discutent, feignent de ne rien remarquer, elles passent un coup de fil puis s'arrêtent à la boutique, la voiture des hommes ralentit, ils vont descendre à leur suite. Pour échanger quelques mots et sortir de l'anonymat, le jeune homme offrira peut-être à la jeune femme une brique de lait ou un soda, initiative qu'elle aura plus ou moins encouragée. Quelques indifférences et quelques œillades plus tard, le ballet reprend. C'est un autre carrefour, une autre boutique, un autre regard... Elle, elle arbore un de ses beaux voiles, qu'elle laisse plus ou moins glisser. Lui, il porte son boubou et parfois son turban [hawli]. Elle a l'esprit d'initiative, elle décide de l'itinéraire. Lui maintient le cap sur sa voiture. Elle décide de la longueur de la promenade, de ses arrêts. Il ne rentrera pas tant qu'il est susceptible de la recroiser ${ }^{32} \ldots$

Dans ces rapports de séduction, diurnes ou nocturnes, l'homme n'a ni l'initiative, ni la force, il doit feindre de se soumettre au désir des femmes 
auquel son propre désir est soumis. Ces «inversions » des rapports de genre prennent place dans des contextes de séduction. Lors de ces réunions extrêmement fréquentes, les femmes, sur l'asphalte ou sous la tente, ne sont plus celles qu'elles devraient être et ce sont les normes englobantes sur le rapport de genre qui évoluent avec elles. Dans ces circonstances, elles ne sont plus les femmes «délurées », " chaudes », incapables de mettre un frein à leurs besoins sexuels. Il n'est pas question non plus de folie. Au contraire, la maîtrise de leur désir - et leurs capacités à simuler la maîtrise, ou le désir lui-même - est telle qu'elles peuvent en jouer, flirter, encourager les hommes à s'approcher, à discuter, et parfois même à les toucher car elles savent le manque de maîtrise masculin. N'est-ce pas d'ailleurs pour cette raison qu'ils sont là, si nombreux? Et les hommes, en de tels instants, sont également conscients de cette capacité féminine, ce qui leur permet, très certainement, de moins veiller au contrôle de leurs propres pulsions. Chacun joue, ensemble, plus ou moins bien son rôle: l'homme doit se montrer séduisant séducteur (djabbab), jouer parfois l'incapacité de maîtriser son désir pour prouver aux yeux de tous qu'il est un homme. Les femmes s'ingénient à mettre en scène leurs désirs, les dire, les cacher, feindre leur contrôle ou leur non contrôle. Mais elles se savent puissantes: «Jusqu'où irez-vous pour moi? »

\section{Convertir son capital}

La beauté des femmes et leurs pouvoirs de séduction les exposent inévitablement aux convoitises masculines, convoitises qui conduisent à divers types - combinables ou non - de relations hommes/femmes. Pour quelques-unes, la beauté permet un beau mariage, une union avec un homme riche et puissant. D'autres emploient leur beauté à flirter avec les générosités masculines. Ces amours, canalisées ou dispersées, mais toujours lucratives, permettent à certaines femmes d'acquérir des biens parfois considérables dont elles peuvent profiter simplement ou qu'elles peuvent réinvestir dans le commerce ${ }^{33}$.

«Mokeltoum était l'une de ces femmes. Réputée pour son incroyable sourire, son port altier, son bon goût, plutôt mince mais aux formes généreuses, les adolescents filaient la très charmante baydhâniyya dans les ruelles ensablées de Chinguetti ${ }^{34}$ dans

31. Le Bana Blanc est une grande boutique d'alimentation, le Prince un snack très prisé. Ce jeu de show-off urbain a également été relevé par A. Choplin, «Fabriquer des villes-capitales entre monde arabe et Afrique noire. Étude comparative Nouakchott (Mauritanie) et Khartoum (Soudan) », thèse de doctorat en géographie, Université de Paris 1, 2006.

32. Notes de terrain, février 2004.

33. Au sujet des femmes commerçantes de Mauritanie, citons l'ouvrage de G. Simard, Petites commerçantes de Mauritanie. Voiles, perles et henné, Paris, ACCT, Karthala, 1996.

34. Ville située près d'Atar, dans la région de l'Adrar. 
l'espoir d'apercevoir par transparence, sous un rayon de soleil opportun, les charmes dissimulés sous ses voiles. La splendide Mokeltoum marque encore la mémoire des gens de l'Adrar et de ceux qui l'ont connue à Nouakchott ou Nouadhibou. Et pourtant Mokeltoum est décédée voici plus de quarante ans...

Dans les années 1940, à peine âgée d'une vingtaine d'années, elle épouse un riche Français installé en Mauritanie pour affaires. Il a trente ans de plus qu'elle, il est fortuné, il n'est pas musulman. Elle est magnifique et elle n'en est pas à sa première aventure. L'union soulève la désapprobation des anciens et on se souvient encore du scandale et des pourparlers qui entourèrent cette union. Mais, comme s'accordent à dire des "parents" de la jeune femme, "ces années étaient difficiles" : les effets de la Seconde guerre mondiale et de la défaite française, la lourdeur de la politique coloniale (réquisitions, rationnements...) ainsi que de mauvaises conditions climatiques plongent la région de l'Adrar dans une misère noire: "C'était la disette", "tout le monde avait faim". Marier Mokeltoum à l'étranger a permis à la famille de traverser cette période de grave crise économique: "[Mokeltoum] était livrée à elle-même et devait subvenir à ses besoins", "elle était à la merci du premier venu", "un sauveteur était le bienvenu". Le marié a pourvu abondamment aux besoins de toute la famille - au sens élargi - jusqu'à ce que Mokeltoum, sponsorisée par son époux, se lance dans le commerce d'importation et devienne en quelques années une femme d'affaires puissante, capable d'aider les siens. »

D'autres femmes en usant des bontés de la nature ont profité de soutiens épars, transformant ainsi leur capital beauté en capital économique.

" $\mathrm{D}^{\prime}$ une mère Aqzâzir ${ }^{35}$ et d'un père Hartâni (affranchi) de la tribu des Smâsîd, Lala est née à Atar, au début des années 1930, dans le quartier Camp d'Amérique. La jeune fille ne suit pas l'école. À 15 ans, elle est donnée en mariage à un cousin. Le couple s'installe d'abord à Nouakchott, mais après leur divorce, Lala décide de partir pour Thiès, "car il y avait beaucoup de Mauritaniens qui voulaient faire fortune au Sénégal", témoigne sa fille adoptive. Après une période qualifiée de "floue" par un membre de sa famille mais d'errance voluptueusement lucrative selon d'autres témoins, Lala devient la compagne d'un homme peu fortuné mais de grande famille. Du fait de ses origines sociales et des "flous" de sa biographie, leur union fait quelque peu scandale. Mais Lala s'en moque: avec ses propres économies, et un petit apport de son mari, elle ouvre un bazar où sont vendus tissus et biens d'alimentation... L'affaire réalise quelques bénéfices.

En 1960, à l'indépendance de la Mauritanie, le couple choisit l'option du retour et s'installe à Nouakchott. Lala se relance dans le commerce et ouvre une boutique "dans un quartier chic de toubabs [Européens]". Très vite, les compétences relationnelles de la jeune femme (charme, humour, sens de la poésie et des affaires) tissent autour du ménage un important réseau de sociabilité regroupant des commerçants grossistes, des négociantes, des fonctionnaires, des représentants des autorités locales: " $C$ 'était une femme d'une forte personnalité, elle participait au débat des hommes, pour discuter de la politique mais aussi pour rivaliser avec ses poésies. Elle avait beaucoup de relations car tout le monde l'appréciait, et aimait sa compagnie", rapporte sa fille, qui ajoute que "son mari était toujours jaloux car tous les hommes étaient amoureux". L'entreprise connaît une croissance très rapide, laquelle conduit Lala à acheter de nouvelles boutiques et multiplier ses voyages de ravitaillement en marchandises (en Afrique de l'Ouest, en Europe et en Arabie Saoudite).» 


\section{Se (re)placer sur l'échiquier}

Les jeux enchanteurs de ces splendides stratèges, de ces plantureuses opportunistes, les conduisent à une fortune dont la redistribution leur permet de conquérir une nouvelle place dans la hiérarchie sociale. Ainsi, dans la mémoire collective, Mokeltoum n'est pas une Cendrillon redevable à son prince charmant, mais avant tout une notabilité à la générosité presque légendaire, une pourvoyeuse d'emplois, une militante politique active, engagée humainement et financièrement derrière Mokhtar Ould Daddah, premier Président de la Mauritanie, une épouse dont l'influence, sociale et économique, aurait même permis à son bienfaiteur mari français d'être élu conseiller territorial.

Mais peut-être plus encore que la richesse ou sa redistribution, ce sont la combativité et les tours de force mobilisés, les artifices, l'intelligence, la bravoure, la capacité à jouer de ses avantages qui permettent à certaines femmes de (re)négocier leur place dans la société ${ }^{36}$.

Lala est considérée comme une grande commerçante, comme une femme incontournable dans la vie mondaine des années 1960. Pourtant Lala est partie de rien : ses premiers profits sont dus à des cabotages amoureux; ses relations sociales ne sont pas héritées mais tissées à partir de son charme, de son humour, de son intelligence. Issue d'un groupe considéré au bas de l'échelle sociale, le triomphe de Lala ne l'a évidemment pas anoblie mais il lui a permis de se frayer une place dans la société plus importante que celle que ne le laissait sans doute augurer sa naissance. Aujourd'hui, quelques années après son décès, Lala est toujours appelée par son surnom, lequel fait référence à ses origines sociales: «ça la faisait rire, c'étaient les commerçantes jalouses qui l'ont inventé et elle a voulu le garder», mais il est tout autant fait mention de son esprit vif, de ses plaisanteries, de ses atours, de ses extravagances, de ses voyages, de sa générosité, de ses boutiques imposantes et du rôle qui était le sien dans la vie nouakchottoise ${ }^{37}$.

Si l'art de la beauté peut servir à renégocier une position hiérarchique, via par exemple la réussite commerciale, il peut propulser quelques Dames au sommet plus rapidement encore. Le messrah, en arabe et en hassâniyya, désigne littéralement la scène et, par extension, le théâtre. Pour les Mauritaniens,

35. Un groupe de saulniers, considéré comme étant au bas de la hiérarchie sociale.

36. Sur l'importance des manœuvres individuelles pour expliquer les parcours de réussite, nous adhérons aux analyses de Z. Ould Ahmed Salem développées dans «Tcheb-tchib et compagnie. Lexique de la survie et figures de la réussite en Mauritanie», Politique africaine, $\mathrm{n}^{\circ}$ 82, juin 2001, p. $78-100$.

37. Sur le Nouakchott des années 1960-1970, voir E. Ould Mohamed Baba, De mémoire de Nouakchottois, chroniques du temps qui passe, Paris, L'Harmattan, 2004, p. 12. 
"être sur le messrah» signifie donc «être sous les feux de la rampe», ou, en d'autres termes, appartenir à une certaine élite. On dit de ces gens du messrah, qu'ils «font le théâtre », qu'ils «s'affichent», qu'ils "vont paraître ", qu'ils «brillent» - cette dernière expression étant surtout utilisée pour les femmes, alors désignées sous le terme de $m c h a^{\prime} c h^{\prime} a, l^{\prime}$ «étincelante», la «flambeuse». Parce que le messrah impose un genre de vie où le luxe est la règle des concurrences, nombreux sont ceux qui, dotés d'un capital, aspirent à égaler les fastes de cette élite-modèle. Pour copier cette jet set, pour être accepté par elle, chacun doit relever des défis et prouver par la dépense ses titres au prestige ${ }^{38}$... C'est dans cette économie de l'ostentation que se crée la distinction sociale, «que la hiérarchie se bâtit ${ }^{39}$ ».

Avoir son nom au générique des spectacles du messrah exige un portefeuille solide pour les hommes, et des attraits très vifs pour certaines femmes... Les membres masculins de cette jet set appartiennent à «la classe politicocommerciale $40 »$ : hauts fonctionnaires, banquiers, businessmen, anciens ministres... Ses membres féminins sont, d'une part, les charmantes épouses des hommes susmentionnés (souvent par ailleurs engagées dans le commerce) et d'autre part, quelques dames de charme dont l'activité principale consiste à être en beauté: «ce sont des princesses, elles se lèvent à 17 heures, se préparent et vont aux invitations ${ }^{41}$ ». Dans les deux cas, ce sont les qualités esthétiques et leur usage (mariage ou badinages) qui ouvrent les portes du messrah.

«Considérée par beaucoup comme touarègue, Aïcha évoque avec nous des parents baydhân. En revanche, sa beauté fait l'unanimité. Aïcha est réputée être la plus belle Nouakchottoise des années 1990. On raconte qu'elle était initialement installée à Abou Dhabi puis à Dakar et c'est alors que tout aurait débuté: "elle avait un gorjigéen 42 qui, à l'aéroport, proposait aux riches Mauritaniens de venir prendre le thé... Et puis, au début des années 1991, comme ça, Monsieur X, un haut fonctionnaire est devenu son amant régulier, elle est venue à Nouakchott [...]".

À son arrivée en Mauritanie les nouvelles vont vite. Aïcha est déjà connue de tous. On raconte. On affirme: "elle est belle" ; "elle se maquille à la perfection" ; "elle est assez mince" ; "elle accepte d'être la maîtresse d'un homme, sans se cacher" ; "elle serait touarègue"; "elle est la bonne amie d'un batrou”. D'emblée, sur sa réputation, Aïcha devient la shabîbâ de la ville et accède au messrah.

Aïcha sort peu. Elle reçoit chez elle avec une parcimonie judicieuse et fructueuse. Elle s'entoure de mystères, et c'est sans doute la raison pour laquelle elle se maintiendra sous les feux de la rampe pendant presque dix années. Les hommes qui ont eu le privilège d'être ses invités vantent ses qualités de poétesse, son hospitalité: "Il n’y a aucune femme de la place qui reçoit comme elle sait le faire. Elle connaît les hommes qui viennent chez elle, elle sait ce qu'ils veulent entendre, elle sait les flatter, les mettre à l'aise. Elle est aux petits soins, comme on dit [...]. Quand on est chez elle, on se sent le centre du monde", témoigne un habitué des mondanités. Dans le luxe de son salon, on boit le thé. Un homme, ou plusieurs en concurrence. Elle siège parmi eux. Elle est 
objet de convoitises, elle est couverte de cadeaux, de compliments. Le flirt est l'enjeu principal de cette invitation. Quoi qu'il en coûte à ces messieurs. Le dialogue, du verbal, peut passer au corporel, au bon vouloir de la jeune femme, réputée sélective dans le choix de ses amants.»

De l'avis de nombreux Mauritaniens, Aïcha est une des plus grandes batrounnes et cette pimpante réussite ainsi que l'étendue de ses relations en haut lieu n'autorisent pas à de réelle discréditation de façon publique. Cependant on ne s'abstient pas de murmurer qu'elle a été une femme aux mœurs légères. Quelques commerçantes suggéreront qu'elles n'ont pas le même parcours. Des Mauritaniens chuchoteront que l'amoureuse dispersée est venue de nulle part. Aïcha (tout comme Lala) n'est pas issue de l'aristocratie traditionnelle, ses entorses à la bienséance morale sont donc plus difficiles à faire taire. Personne n'oublie le passé. Cependant grâce à son ascension vertigineuse, Aïcha est tout autant considérée comme une femme qui s'est affirmée, une femme qui a réussi, une femme rusée qui a su instrumentaliser les opportunités qu'offre un joli minois. Parce qu'aux yeux de tous elle a particulièrement bien joué et parce qu'elle a considérablement gagné, Aïcha campe, dans l'imaginaire social, le premier rôle féminin d'une prestigieuse épopée affairiste. Une star qui fait même rêver quelques jeunes filles car Aïcha est une figure marquante du messrah, elle appartient à ce groupe important et cette affiliation lui confère

38. Ces compétitions contemporaines ne sont pas sans rappeler les traditionnels vaysh (défis), joutes productrices de classements. L'identité d'un(e) homme/femme s'inscrit certes en fonction de ses positions de parenté, statutaires, politiques mais aussi dans une dynamique incessante de différenciation classificatoire dans laquelle la quête de l'honneur demeure le principal moteur. Ces «contestations d'honneur» ont traditionnellement pour support des biens de prestige : les chevaux, la beauté féminine, le verbe poétique ou tout «autre bien qui se trouve dans le harim de l'individu : griots, znâga [tributaire]...». Voir A. Leriche cité par P. Bonte, "L'émirat de l'Adrar. Histoire et anthropologie d'une société tribale du Sahara occidental », thèse de doctorat d'État, Paris, EHESS, 1998, p. 1204. Ces rivalités s'organisent aujourd'hui autour d'objets nouveaux - villas, voyages, voitures, téléphones, et c'est la rumeur qui se charge de faire connaître l'excellence des uns ou des autres. Ces «défis d'essence individuelle » éprouvent les qualités masculines (honneur, générosité, opportunisme) et féminines (beauté, séduction, art de plaire et de converser) et classent les individus sur l'échelle sociale. Voir P. Bonte, «L'émirat de l'Adrar...», op. cit., p. 1208

39. J.-P. Warnier, Approches de la culture..., op. cit., p. 118.

40. J.-F. Bayart, L'État en Afrique. La politique du ventre, Paris, Fayard, 1989.

41. Entretien avec un «mondain», Nouakchott, avril 2004.

42. En langue wolof, góor signifie l'homme et jigéen, la femme. Comme son nom l'indique alors, le góoriigéen est celui qui est homme-femme. Cette métaphore permet de jouer sur l'ambiguïté: en Mauritanie, les góorjigéen sont des hommes qui cultivent une allure efféminée, les faisant passer pour des homosexuels, ce que tous ne sont pas. Ce ne sont pas tant, d'emblée, les pratiques sexuelles, pensées comme anormales par l'ensemble de la société, qui sont désignées sous ce terme mais le non-respect de la limite des genres car cette ambiguïé, entretenue par les hommes-femmes, peut les amener à exercer un certain pouvoir social. 
un statut particulier qui la propulse au sommet d'une hiérarchie sociale nouvelle où l'impératif de la naissance peut parfois s'effacer au profit de la beauté et de la richesse.

D'autres shabîbât ont eu accès au messrah. Ce "privilège», elles en ont usé pour établir de bonnes relations, se faire connaître et reconnaître et pour imposer leur business. Certaines évoluaient déjà dans un petit groupe (surnommé la «mafia d'Akjoujt») composé notamment de femmes de la région d'Atar ou d'Akjoujt, connues pour leur liberté, leur goût de la provocation et leur beauté. À Nouakchott, celles qui étaient issues de bonne famille ont pu accéder au messrah en épousant des membres de la nouvelle élite nouakchottoise ${ }^{43}$. Aujourd'hui, ces femmes sont à la tête d'entreprises commerciales florissantes. Elles remportent les appels d'offre lancés par l'État. Elles soutiennent des candidats aux élections. En pénétrant sur le messrah, les beautés reconverties en businesswomen, accèdent aux univers étroitement liés des affaires et de la politique.

Il en est ainsi notamment de Zeïnabou, plus réputée pour son charme, son caractère et ses affaires que pour sa joliesse. Femme active aux grandes compétences relationnelles, elle perce dans le commerce et pénètre peu à peu le messrah, au tout début des années 1990. Dix ans plus tard, elle est la femme d'affaires la plus connue de Nouakchott, et dirige une association de négociantes qui a fait bâtir son propre centre commercial. Pour la construction de ce marché de femmes, l'association a bénéficié d'un crédit inespéré: «C'est un coup de pub. [...] Les femmes sont le credo du Président [de la République, Ould Taya]. En plus, ce sont des femmes de grandes familles, alors là, c'est pour se faire bien voir ${ }^{44}{ }$. Un homme d'affaires confirme:

«Il s'agit de femmes de la même région que le Président, des femmes puissantes, connues de tous. [...] La banque en était à ses débuts, le Président de la République encourage les femmes [...], le directeur de la banque a saisi le créneau. [...]. C'est une façon de se faire bien voir, de se faire remarquer ${ }^{45}$.»

De très nombreux facteurs interviennent dans cette promotion féminine sélective - et notamment l'instrumentalisation des appartenances tribales et régionales -, mais il est clair que l'un des arguments de ces Dames est celui d'être en vue. Ce sont des femmes qui pèsent et qui veulent peser encore plus. Et pour cela, il peut être de bon augure de mettre le pied dans la sphère politique...

Aux élections législatives de novembre 2006, Ahmed Ould Daddah, chef de file du Rassemblement des forces démocratiques (RFD), a ainsi préféré Zeïnabou à une militante de longue date pour figurer en quatrième position de sa liste; l'adhésion de la commerçante à un parti d'opposition, soudain devenu l'un des partis les mieux placés dans la course au pouvoir, est récente: jusqu'au 
renversement du président Ould Taya par les militaires en août 2005, Zeïnabou s'affichait en effet dans les rangs du parti présidentiel... Pour la santé de leurs affaires, dans la transition démocratique assurée par le Comité militaire pour la justice et la démocratie (CMJD), les femmes du messrah font tout pour compter avec le pouvoir à venir. En simple militante ou si possible en tant que candidate, la course aux opportunismes les invite à choisir, sans transition, un camp officiel. L'ordonnance prise le 22 août 2006 par le CMJD «portant loi organique relative à la promotion de l'accès des femmes aux mandats électoraux et aux fonctions électives » intervient alors à point nommé: cette mesure, entrée en vigueur pour les élections législatives et municipales de novembre 2006, impose désormais des quotas féminins dans les listes électorales, et leur réserve des places définies ${ }^{46}$. Les partis sont donc dans l'obligation d'intégrer plus de femmes et de les mettre en avant. Il leur faut de préférence recruter des femmes qui disposent de ressources financières et matérielles qu'elles pourront mettre au service de la campagne, mais aussi des femmes connues et influentes, capables de séduire l'opinion publique. Les Dames du messrah (entre autres, Zeïnabou) se retrouvent alors en première ligne, comme en témoigne, désabusée, une militante de la société civile:

«Qui vont être les femmes de la politique? [...] Des commerçantes illettrées? Des femmes qui dorment toute la journée? Il n'y a pas un stock de femmes, formées et compétentes, en Mauritanie qui peut prendre les places [proposées]. Qui va vraiment pouvoir faire ça ${ }^{47}$ ?»

Cette ordonnance destinée à la promotion féminine s'adresse-t-elle vraiment à toutes les femmes?

43. Les «mafieuses" non affiliées à l'aristocratie traditionnelle ont eu plus de difficultés à épouser de bons partis. Elles n'ont pu dans l'ensemble percer sur le messrah: les hiérarchies sociales traditionnelles influent donc sur les voies d'accès à la réussite, mais si l'appartenance à un groupe bien positionné sur l'échelle sociale peut-être un atout non négligeable dans les trajectoires professionnelles des femmes d'affaires, elle n'est pas suffisante... Dans une majorité des cas (comme ceux de Mokeltoum, des «mafieuses » de bonne tente ou de Zeïnabou, discuté plus bas), une ascendance noble facilite la mise entre parenthèses d'épisodes amoureux marqués par la «dispersion »; que certains détails soient tus participe alors à créer plus de légitimité, sans que personne ne soit dupe.

44. Entretien avec le cadre de la banque qui a accordé un prêt à l'association, Nouakchott, 2004.

45. Entretien avec un conseiller financier, Nouakchott, 2004.

46. Les règles relatives au quota féminin varient selon les scrutins. Les enquêtes sur les femmes en politique ont été réalisées entre octobre 2006 et mars 2007 dans le cadre d'une recherche sur le (non-) renouvellement des élites politiques et économiques en Mauritanie. Au sujet des femmes dans la sphère politique, lire l'étude de A. Daddah, «Ouverture politique en Mauritanie: la démocratisation sans les femmes?», Nouakchott, Secrétariat à la Condition Féminine, 1994.

47. Entretien avec une militante active de la «société civile», Nouakchott, novembre 2007. 
Souffrir pour être belle, au nom du groupe, peut constituer, pour les femmes les plus appréciées ou les plus opportunistes, une aubaine personnelle. Une source de pouvoir. Butinages charnels, mariages et remariages ainsi que jeux badins de séduction permettent à certaines de convertir leur capital beauté en capitaux économiques et sociaux indispensables à leurs aventures commerciales. Certaines sont devenues des femmes d'affaires. Cette réussite leur permet d'accéder à la fortune et la renommée, ou de les conforter. Mais les bénéfices de ces succès sont beaucoup plus étendus: dans certains cas, ils participent à atténuer les «scandales amoureux» ou permettent également à celles «venues de nulle part» de se repositionner sur l'échelle sociale puisque l'argent et les fracas des triomphes ouvrent parfois autant de portes que la naissance.

La beauté, ses effets financiers et la renommée qui en découle permettent également à celles qui le souhaitent de graviter dans les milieux huppés de Nouakchott, ces milieux qui font l'opinion. On y décroche des contrats, des crédits, on y négocie un mariage ou un marché, on y entretient ses contacts... Il est important d'en profiter et il est essentiel d'y demeurer car on est là au cœur de la classe politico-commerciale, celle par qui les affaires continuent, celle par qui une trajectoire politique peut commencer

Céline Lesourd 